\title{
$\operatorname{micr} \oplus$ biology
}

.

!...Comment...?

Microbiology Comment provides a

platform for readers of Microbiology to communicate their personal observations

and opinions in a more informal way than through the submission of papers.

Most of us feel, from time to time, that other authors have not acknowledged the work of our own or other groups or have omitted to interpret important aspects of their own data. Perhaps we have observations that, although not sufficient to merit a full paper, add a further dimension to one published by others In other instances we may have a useful bit of methodology that we would like to share.

The Editors hope that readers will take full advantage of this section and use it to raise matters that hitherto have been confined to a limited audience.

John Freer

Editor-in-Chief

\section{Yeast cyclic AMP concentration can vary 70-fold}

The sole role of adenosine $3^{\prime}: 5^{\prime}$-cyclic monophosphate (cAMP) in cells of the yeast Saccharomyces cerevisiae has been proposed to be that of a biochemical switch whose concentration directly controls entry into, and probably exit from, stationary phase via regulation of the activity of protein kinase A (7). Such a role for cAMP would be best achieved by having similar, low levels under starvation conditions and similar, bigh levels under growth conditions, with intermediate values excluded since these would give conflicting signals to the cell. By mutating genes involved in the regulation and biosynthesis of cAMP it has been possible to create cells with either very high or very low concentrations of cAMP. Studies of such cells have produced results which are consistent with these suggestions (7).

Two recent papers in Nature $(1,6)$ suggest that cAMP concentration inside yeast cells has an additional function, namely in controlling the overall expression of cyclin (CLN) genes. Since cyclin genes are involved in initiation of the cell cycle at start and have a role in determining cell size at start, this new proposal means that cAMP indirectly couples cell cycle progression to growth (biomass). Such a role would be consistent with cAMP levels showing a wide range of concentrations dependent on external conditions such as growth rate, nature of the carbon source and/or substrate concentration. One of the papers referred to the lack of suitable measurements of intracellular cAMP concentrations in wild-type cells grown under a wide range of conditions (6).

We have grown yeast cells in a variety of media and measured intracellular cAMP concentration during exponential growth (as judged by constant rate of growth of the population and a constant fraction of budded cells). We found that there was an inverse linear relationship between cell density and intracellular cAMP content of cells which varied at least 70 -fold (Fig. 1). Since cells were inoculated into these media to give approximately the same initial cell density, higher cell concentrations reflect greater utilization of substrates and reduced availability of residual nutrients.

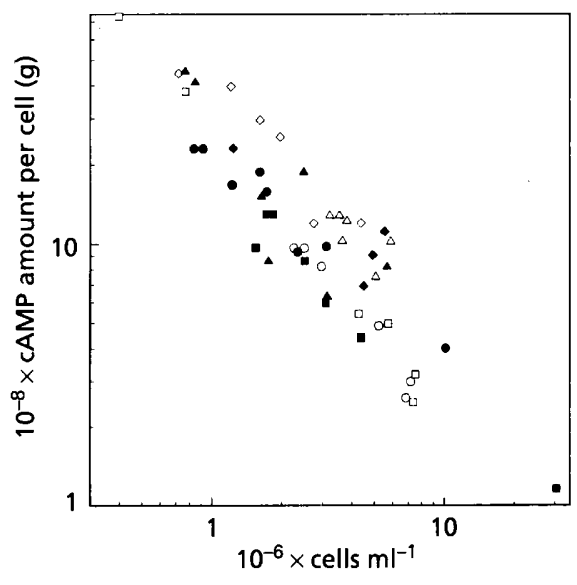

Fig. 1. Intracellular cAMP concentration and cell density in batch cultures. Flasks of complex (YP) or synthetic minimal (S) media (4) containing $2 \%(\mathrm{w} / \mathrm{v})$ D-glucose $(D)$, fructose $(\mathrm{F})$, glycerol $(\mathrm{G})$ or pyruvate (Py), were inoculated with an exponentially growing culture of a diploid prototroph (D1) (5) that had been growing at $25^{\circ} \mathrm{C}$ in SD medium. The cells were then incubated at $25^{\circ} \mathrm{C}$ in a shaking water bath. Samples were taken for cell number and cAMP concentration measurements (2) when the cells had reached midexponential phase (constant fraction of budded cells). $\bullet$, YPD; O, SD; $\boldsymbol{\omega}, \mathrm{YPF} ; \square, \mathrm{SF} ; \boldsymbol{\Delta}, \mathrm{YPG}, \triangle$, SG; $\diamond$, YPPy; $\diamond$, SPy.
Extracellular glucose concentration is known to affect intracellular cAMP concentration (3). Our data suggest that this kind of relationship extends to other carbon sources. Although the correlation between cell density and cAMP differs between fermentable and non-fermentable carbon sources, the difference is less than twofold under otherwise identical conditions. The range of media that were used support a wide range of growth rates but this was not reflected in a consistent relationship to cAMP concentration.

The results are compatible with the earlier biochemical switch model only if cells are particularly sensitive to certain threshold levels of cAMP. The data are fully congruent with the new proposals on the additional role of cAMP but suggest that cAMP concentration continually reflects available external nutrient supply rather than simply growth rate or carbon source as was proposed $(1,6)$. Since it is known that cell size at start is relatively constant at slower growth rates and only increases noticeably at the fastest growth rates, it is likely that the role of cAMP in determining cell size is more complex than has been suggested in either of these papers. To resolve the issue it would be desirable to control independently the level of both cyclin proteins and cAMP, and determine the effect on cell size.

\section{GUIDELINES}

Communications should be in the form of letters and should be brief and to the point. A single small Table or Figure may be included, as may a limited number of references (cited in the text by numbers, and listed in alphabetical order at the end of the letter). A short title (fewer than 50 characters) should be provided.

Approval for publication rests with the Editor-in-Chief, who reserves the right to edit letters and/or to make a brief reply. Other interested persons may also be invited to reply. The Editors of Microbiology do not necessarily agree with the views expressed in Microbiology Comment.

Contributions should be addressed to the Editor-in-Chief via the Editorial Office. 


\section{Alan E. Wheals," 1 Maxine E. Smith' and J. Richard Dickinson ${ }^{2}$ \\ 'School of Biology and Biochemistry, University of Bath, Claverton Down, Bath, BA2 7AY, UK. \\ 2Department of Pure and Applied Biology, University of Wales College of Cardiff, PO Box 915, Cardiff, CF1 3TL, UK.}

*For correspondence. Tel: +44 1225826826 ext. 4278. Fax: +44 1225 826779. e-mail: A.E.Wheals@bath.ac.uk

1. Baroni, M.D., Monti, P. \& Alberghina, L. (1994) Repression of growth-regulated G1 cyclin expression by cyclic AMP in budding yeast. Nature 371, 339-342. 2. Brown, B.L., Albano, J.D.M., Ekins, R.P. \& Sgherzi, A.M. (1971). A simple and sensitive saturation assay method for the measurement of adenosine 3', 5'-cyclic monophosphate. Biochem J 121, 561-562.

3. François, J., Fraso, P. \& Gancedo, C. (1987). Changes in the concentration of cAMP, fructose 2,6-bisphosphate and related metabolites and enzymes in Saccharamyces cerevisiae during growth on glucose. Eur J Biochem 164, 369-373.

4. Sherman, F. (1991). Getting started with yeast. In Guide to Yeast Genetics and Molecular Biology, pp. 3-21. Edited by C. Guthrie \& G. Fink. London: Academic Press. 5. Smith, M., Dickinson, J.R. \& Wheals, A.E. (1990). Intracellular and extracellular levels of cyclic AMP during the cell cycle of Saccharomyces cerevisiae. Yeast 6, 53-60. 6. Tokiwa, G., Tyers, M., Volpe, T. \& Futcher, B. (1994). Inhibition of G1 cyclin activity by the RAS/cAMP pathway in yeast. Nature 371, 342-345.

7. Uno, I. (1992). Role of signal transduction systems in cell proliferation in yeast. Int Rev Cytol 139, 309-332.

\section{Paper Commentary}

\section{Oxidase names: to ' 3 ' or not to '3'?}

In an earlier issue of Microbiology, we presented data on the spectral kinetic properties of a terminal quinol oxidase of Escherichia coli (7). This oxidase in particular has become the subject of intense interest, especially from bioenergeticists, biochemists and spectroscopists who recognize the attractions of a bacterial oxidase that serves as an amenable model for the cytochrome $c$ oxidase (EC 1.9.3.1) of eukaryotes, an enzyme of enormous complexity and difficulty. In that paper, we suggested that due care be given to the nomenclature of this and other bacterial oxidases. The father of cytochrome studies, David Keilin, certainly held this view (3), writing: 'As the position of absorption bands of cytochrome observed in intact cells of microorganisms show certain variations, it is important when referring to these bands to use as uniform a terminology as possible'. However, there are now appearing in the burgeoning literature (10) names that are not only unrecognized in current official nomenclature recommendations but which, in our view, are etymologically inaccurate. The purpose of this commentary on our own paper is to expand the argument advanced there, point out the pitfalls of extending the 'new', unofficial nomenclature to other cytochromes (which seems to be happening) and to draw the attention of the community studying these enzymes to the proper historical background.

\section{The discovery of 'cytochrome 0 '}

In 1954, Lucile Smith, LaRoy Castor and one of us (B.C.) discovered a photodissociable carbon monoxide compound in several types of bacteria, the absorption bands of which were similar to haemoglobin. In view of this similarity, and recognizing the claim by Keilin \& Tissière of the presence of haemoglobin in certain micro-organisms, functional studies were made. Using an improvement of Warburg's photochemical method, Castor \& Chance (2) showed the mysterious haemoprotein to be an oxidase and named it cytochrome $o$. It is important to recognize that

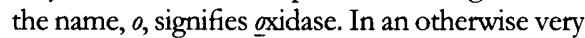
clear summary of haem nomenclature (1), an explanation of 'cytochrome $o$ ' illustrates the present misunderstanding of the name's origin: 'The original designation of an 0 -type cytochrome meant that it contained a heme $B$, which can bind to carbon monoxide (or $\mathrm{O}_{2}$, extrapolating for oxidases)'. Firstly, the name was intended to describe function ( $o$ for oxidase), not haem type. At the time, haem $\mathrm{O}$ (see below) had not been discovered but the spectral similarity with haem B (5) justified the supposition that the ligandreactive haem was $B$. Secondly, this description implies that the characteristic of cytochrome $o$ is $\mathrm{CO}$ binding, whereas Chance \& co-workers coined the name only after the oxidase function, i.e. the reaction with oxygen, was demonstrated by the light reversibility of inhibition of respiration (2). The direct demonstration of the binding of oxygen with cytochrome $o$ to give a compound spectrally resembling the $\mathrm{CO}$ compound was obtained by us only much later (6), with the availability of low temperature photolysis methodologies.

\section{A new era of oxidase research.....}

The explosion of interest in research on bacterial oxidases is largely the consequence of molecular cloning of structural genes, purification of oxidase complexes and the recognition of a much greater diversity of oxidase types than had hitherto been suspected. In addition to the long recognized existence of oxidases containing cytochromes $o, a_{1}, a_{3}, d$ and $d_{1}$ as ligand-binding haems, it has become clear that cytochrome $b$ can act in this capacity and that certain oxidases contain previously unrecognized haem types. For example, the cytochrome 0 -type oxidase of Escherichia coli is now known to contain a novel haem, haem $O$ (8). To add to the possibility of confusion (or interest, depending on one's point of view) it is now known that a haem site in an oxidase protein can be occupied by more than one class of haem. For example, in the cytochrome o-type oxidase of $E$. coli, a variant of the oxidase is known in which both haems are the O-type, in contrast to the 'normal' oxidase in which one haem is $\mathrm{O}$ (ligand-binding) and the other is B (9).

Quite naturally, those working on these oxidases have sought a way of distinguishing, in the name, between haems in a given oxidase. It is particularly valuable to demonstrate in the name which of the haems is, or are, capable of binding oxygen (the substrate) and other ligands such as $\mathrm{CO}$ and cyanide. Precisely this requirement arose in the 1930s, when Keilin (3) described 'two...compounds having the same haem nucleus. One of these compounds $(a)$ is not autoxidizable and does not combine with potassium cyanide or carbon monoxide, while the other $\left(a_{3}\right)$ is autoxidizable and combines with both'. Why was the name $a_{3}$ chosen? Again, Keilin himself makes this perfectly clear. 'We shall designate this new component as $a_{3}$ in order to distinguish it from cytochromes $a_{1}(589 \mathrm{~nm})$ and $a_{2}(630 \mathrm{~nm})$ which are known to occur only in a few bacteria devoid of cytochrome a.' (We know now that certain bacteria do possess cytochrome $a_{3}$ as well as ' $a_{2}$ ', but the principle of nomenclature remains valid.) Cytochrome $a_{1}$ was the name given to a cytochrome observed in Acetobacter and a few other bacteria. This name is now little used because in many bacteria, but not all (10), cytochrome $a_{1}$ has been shown to be a high-spin $b$-type cytochrome that is a component of the cytochrome bd complex. Cytochrome $a_{2}$ was the early name for cytochrome $d$. Like most types of ' $a$ ', it was subsequently shown not to contain haem $\mathrm{A}$ but, in this case, a chlorin haem that gives rise to the unique spectral features of this type of oxidase.

\section{....but do we need new names, and are some of them really new?}

It is with supposed deference to the name ' $a a_{3}$ ' that the names $b o_{3}, b b_{3}$ and others have been adopted by some workers in this field over the past few years (for a survey, see 10). The subscript ' 3 ' denotes the ligand-binding haem. The name was first used for the $E$. coli cytochrome o-type oxidase by Puustinen \& Wikström (8), who suggested that this oxidase be called $b_{3}$ (when the haems are $\mathrm{B}$ and $\mathrm{O}$ ) or $\mathrm{OO}_{3}$ (when both haems are $\mathrm{O}$ ). It is claimed that the nomenclature is useful because it shows the haem types (we agree), that it shows which is the ligand-binding haem (it does, but a system for doing so already exists, see below), and that it is 'historically pertinent' (we disagree). If ' $a_{3}$ ' has the same etymological root as $a_{3}$, where are cytochromes $o_{1}$ and $o_{2}$ ?

We accept that the subscript is useful in signifying which haem binds oxygen. This is a particularly valuable feature in cases like cytochromes $o o$ and $b b$ where the low-spin (electron-conducting) and high-spin (ligandbinding) haems are of the same chemical structure differing only in their axial ligation to the protein and thus their ability to bind oxygen. However, there exists already an internationally validated recommendation (4) that serves precisely this function. It is simple and unambiguous, namely that the 5-coordinate ligandbinding haem is primed as in $b o^{\prime}$ (for the E. coli oxidase) and $a a^{\prime}$ (for the mitochondrial oxidase).

Unfortunately, this nomenclature has never 'caught on' amongst students of the mitochondrial oxidase, probably because the official recommendation lagged many years behind the 